\title{
Deep Learning as a Tool for Image Denoising and Drift Correction
}

Rama K. Vasudevan ${ }^{1,2^{*}}$ and Stephen Jesse ${ }^{1,2}$

${ }^{1}$ Center for Nanophase Materials Sciences, Oak Ridge National Laboratory, Oak Ridge, TN, USA.

2. Institute for Functional Imaging of Materials, Oak Ridge National Laboratory, Oak Ridge, TN, USA.

* Corresponding author: vasudevanrk@ornl.gov

Drift of the microscope during imaging is an unavoidable and generally undesired attribute that impedes high quality imaging and spectroscopic studies. As a result, means for compensating drift have been developed $[1,2]$ and utilized by the community for several decades. In some cases, the methods require knowledge of the crystal structure as inputs to the algorithm. Regardless, continual improvement in drift correction, especially during microscope operation, will be needed to make studies such as recent atomic manipulation efforts [3] possible at larger scales.

Recently, it has been shown that deep convolutional neural networks (DCNN) offer a remarkable ability to automatically locate and classify atomic-scale defects in images captured from scanning tunneling and scanning transmission electron microscopy $[4,5]$. The remarkable success of DCNNs is likely due to the fact that such methods are often more robust against noise than traditional feature-crafted methods [6], as the deep networks are composed of layers of increasingly more abstract learned features.

Here, we explore whether DCNNs can be useful to quickly diagnose and rectify drift issues in the microscope. As a proof of principle, we operate entirely on simulated data of a hexagonal atomic lattice with different lattice spacings and rotation. We simulated these hexagonal lattices with the addition of uniform noise of a differing intensity. We then took the same image set and applied a simple displacement transformation of known magnitude, thus resulting in two images: one before and one after the linear transformation (see Fig. 1(a,b)). The goal of the network was to learn the transformation, represented as a simple $[\mathrm{x}, \mathrm{y}]$ vector.

Our approach consisted of three distinct steps: (1) Denoise the two images using a specifically trained DCNN, (2) Calculate the normalized cross correlation between the two denoised images, and (3) Use another DCNN on the cross-correlation matrix to learn the transformation. The reasons for using cross correlation as an input is that we found empirically that simply inputting the two images is insufficient for the network to learn the appropriate mapping. This is probably because CNNs utilize spatial invariance due to the convolution operation, whereas in this example, our goal is specifically the opposite, i.e. to learn a spatial transformation. Moreover, calculating the normalized cross correlation between the two images is more accurate if the images are first denoised. We trained a separate DCNN to accomplish the denoising, and an example output of running the two images in Fig. 1(a,b) through this network are shown in Fig. 1(c,d). Based on this, we calculate the normalized 2D Cross correlation, and then input this to our DCNN, whose architecture is shown in Fig. 1(e), and trained to learn the transformation. The results of training the DCNN are shown in Fig. 1(f). In total we trained for 250 batches, with each batch consisting of 1024 image pairs (giving a total of 256,000 image data pairs). As Fig. 1(g) shows, there is a steady increase in the accuracy of the network as training progresses and giving a total validation accuracy of just above $80 \%$. Further results on the validation set for the $x$ and $y$ shifts of the transformation are shown in Fig. $1(\mathrm{~g}, \mathrm{~h})$. Note that this is calculated based on the error between the predicted transformation vector and the actual transformation vector. Efforts are ongoing at 
(a) generalizing to more realistic situations where defects and voids are present, and (b) implementing this setup on an electron microscope [7].

\section{References:}

[1] L Jones and PD Nellist, Microsc. Microanal. 19 (2013), p. 1050.

[2] X Sang and JM LeBeau, Ultramicroscopy 138 (2014), p. 28.

[3] O Dyck et al., Appl. Phys. Lett. 111 (2017), p. 113104.

[4] M Ziatdinov et al., ACS Nano 11 (2017), p. 12742.

[5] M Ziatdinov et al., npj Comp. Mater. 5 (2019), p. 12.

[6] Y LeCun, Y Bengio and G Hinton, Nature 521 (2015), p. 436.

[7] This work was conducted at and supported by the Center for Nanophase Materials Sciences, which is a US DOE Office of Science User Facility.
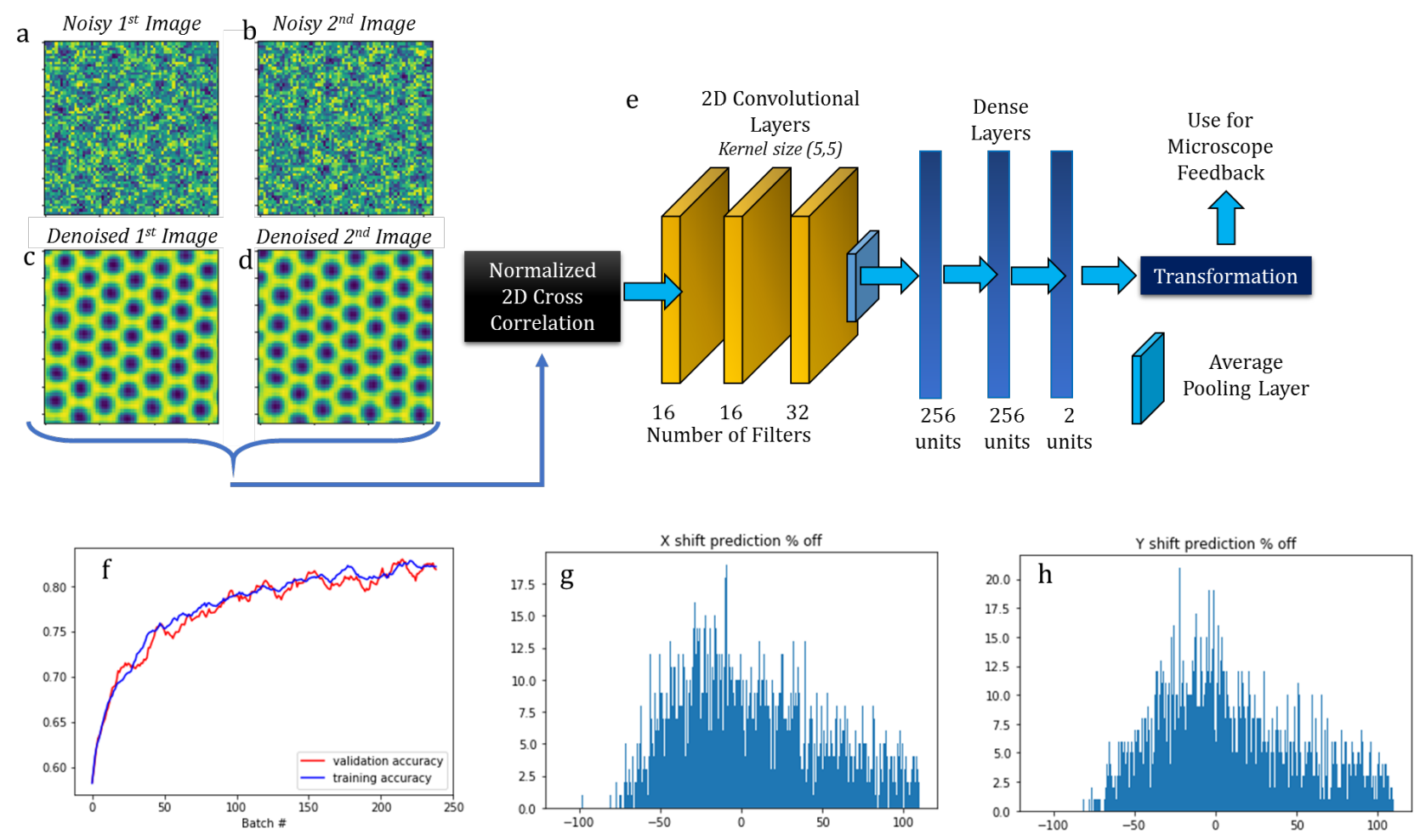

Figure 1. Deep Learning for Image Denoising and Drift Correction. (a,b) Simulated images of a hexagonal lattice with the image in (a) shifted by some amount and plotted in (b). The aim is to learn the level of shifts. To accomplish this, the images are first denoised, via a separate deep convolutional neural network, with results shown in $(\mathrm{c}, \mathrm{d})$. The normalized 2D cross-correlation is calculated and fed to the DCNN in (e), which consists of several convolutional layers followed by three fully connected layers. This then outputs the transformation (i.e., the image shift). The training of this DCNN is shown in (f) as a function of batch number. Results are on the validation set are shown in (g,h). 